\title{
Planetary nebulae: 20 years of Hubble inquiry
}

\author{
Bruce Balick \\ Dept. of Astronomy, University of Washington, \\ Box 351580, Seattle WA 98195 USA \\ email: balick@uw.edu
}

\begin{abstract}
The Hubble Space Telescope has served the critical roles of microscope and movie camera in the past 20 years of research on planetary nebulae ("PNe"). We have glimpsed the details of the evolving structures of neutral and ionized post-AGB objects, built ingenious heuristic models that mimic these structures, and constrained most of the relevant physical processes with careful observations and interpretation. We have searched for close physical binary stars with spatial resolution $\sim 50 \mathrm{AU}$ at $1 \mathrm{AU}$, located jets emerging from the nucleus at speeds up to $2000 \mathrm{~km} \mathrm{~s}^{-1}$ and matched newly discovered molecular and X-ray emission regions to physical substructures in order to better understand how stellar winds and ionizing radiation interact to form the lovely symmetries that are observed. Ultraviolet spectra of CNO in PNe help to uncover how stars process deep inside AGB stars with unstable nuclear burning zones. HST broadband imaging has been at the forefront of uncovering surprisingly complex wind morphologies produced at the tip of the AGB, and has led to an increasing realization of the potentially vital roles of close binary stars and emerging magnetic fields in shaping stellar winds.
\end{abstract}

Keywords. planetary nebulae: general, instrumentation: high angular resolution

I can vividly remember having coffee on a terrace with other attendees at IAU Symposium 155 on planetary nebulae ("PNe") in Innsbruck in 1992, about two years before the first eye-popping Hubble Space Telescope ("HST") image of a PN was released. Several exciting talks on the first two-dimensional hydrodynamic models had just been presented that very successfully matched the morphologies and kinematics of simple PNe (as they were then understood). Someone - probably this author - said that PNe were now basically understood, our project was finished, and now it was time to take the hydro models and apply them to other types of outflow nebulae such as AGNs. How utterly wrong!

By 1994 Hubble images of young stellar objects, $\eta$ Carina, Orion, SN1987a, AGNs, and other nebulae had enthralled everyone. So the community awaited the first image of a PN with high expectations. This paper is a review of the observational highlights of HST observations since then.

The first high-quality Hubble image of a PN, NGC6543, was released in January 1995 (Harrington \& Borkowsky, GO5403; Fig. 1, left)†. I recall both the excitement and the astonishment of this event. The micro-scale complexity of the structure was stunning. A minute later elation morphed into the sobering realization that many years of challenging theoretical work would be necessary before we could claim to understand how PNe form and evolve. We're still on that quest, even today. The progress has been wholly satisfying. (We all know that HST images also upended this sort of premature confidence in many other fields of astrophysics and, so, opened new vistas.)

$\dagger$ NGC6543 was not the first PN to be imaged by Hubble. An FOC image of N66, a PN in the LMC, was obtained in 1991 and released in 1992 by C. Blades and the FOC team. However, N66 is just 2.4" in size and its image was compromised by the quality of the HST optics at the time. So the image of N66 had far less impact than that of NGC6543 released four years later. 


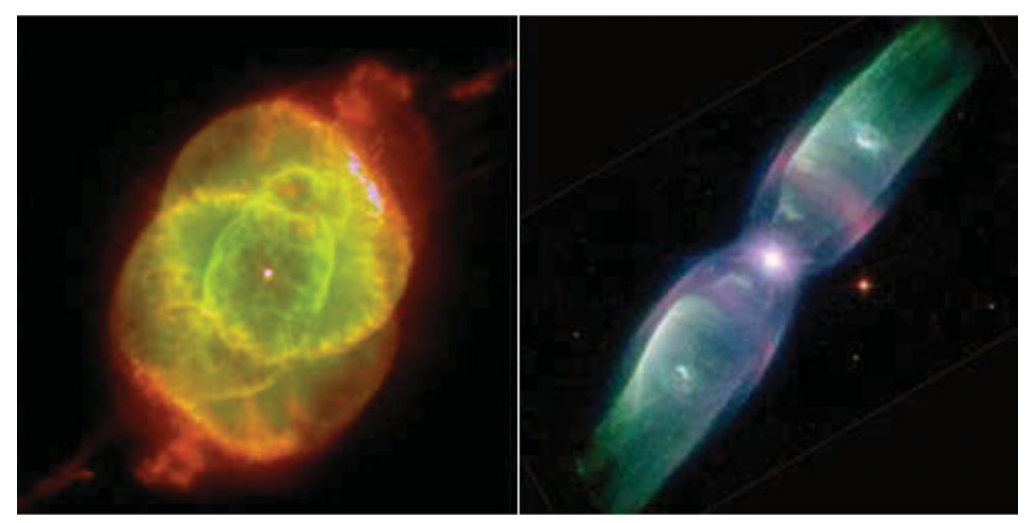

Figure 1. Iconic images of PNe obtained with HST early in its history. Left: NGC6543 (the "Cat's Eye") by P. Harrington \& K.J. Borkowsky (GO5403, images obtained in 1994). Right: M2-9 (the "Butterfly") by B. Balick \& V. Icke (GO6502, images obtained in 1997). The colors are synthesized from images in various narrowband filters. (Color version online only.)
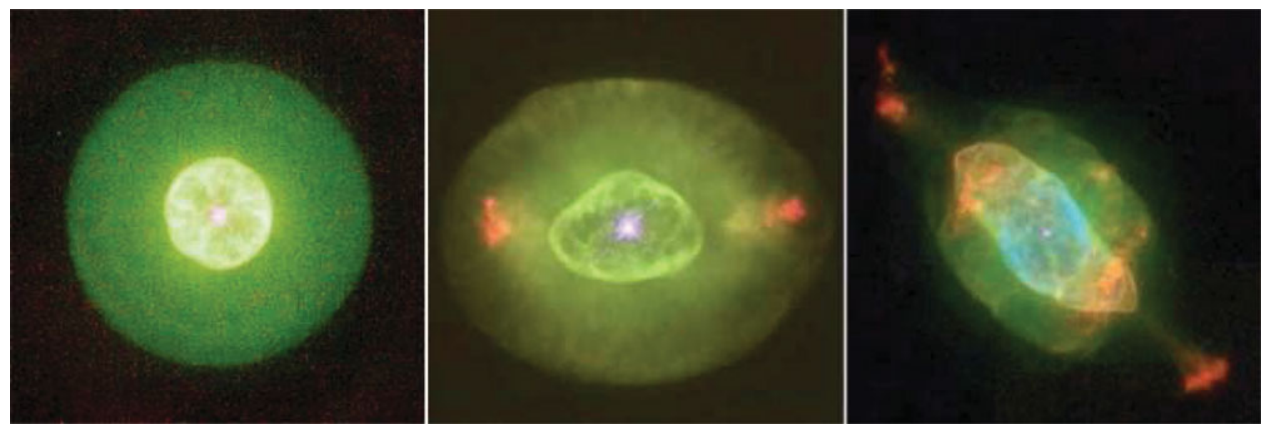

Figure 2. Early HST images of PNe. From left to right: IC3568 (round), NGC6826 (mild elliptical), and NGC7009 (extreme elliptical). Red, green, and blue colors represent the emission from $\mathrm{N}^{+}(\mathrm{F} 658 \mathrm{~N}), \mathrm{O}^{++}(\mathrm{F} 502 \mathrm{~N})$, and $\mathrm{He}^{++}(\mathrm{F} 469 \mathrm{~N})$, each of which arises in complementary volumes of the nebula. These WFPC2 images illustrate three of the basic morphological types of PNe. The small red features in the latter two images are "low-ionization structures" whose formation and persistence are only poorly understood. (Color version online only.)

HST images of many more PNe were soon to follow. H. Bond and R. Ciardullo (GO6119) had used wideband filters and very short exposures to survey a large number of PNe for resolvable binary central stars. They began reporting the new PN images at meetings by 1997. (Their results appeared in Ciardullo et al. 1999.) In December 1997 color images of seven more PNe appeared in a NASA-HST press release (1997-38). Three of these images are shown n Fig. 2. The image set also included the first bipolar nebulae M2-9 (Fig. 1, right) and Hubble 5. NASA-HST press releases of PNe are now routine.

The images in Fig. 2 illustrate the basic features of round and elliptical PNe. These are a central bubble (sometimes filled with soft X-rays) surrounded by a bright and generally thin rim situated within a more extended and amorphous shell with a sharp leading edge. The origin and evolution of these features are now being very successfully reproduced with sophisticated one-dimensional radiation hydro models by R. Jacob, D. Schönberner, and their colleagues. The HST images provided the essential clue, to wit., the sharpness of the leading edges of the rims and shells can be explained only by shocks at the interfaces of regions of very different pressures. Similarly, the small low-ionization (red) structures seen in several PNe like NGC6826 and 7009 seem to require at least converging flows, if 


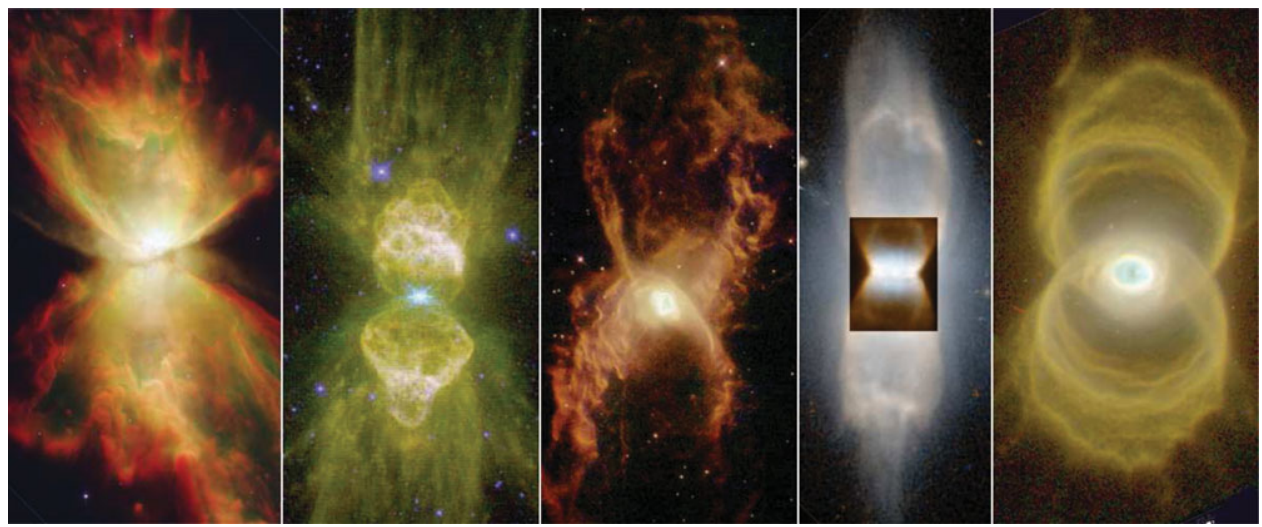

Figure 3. A montage of bipolar PNe with pinched waists - a morphology that hints that the outflows in the lobes are collimated in close proximity to their sources. From left to right the nebulae are NGC6302, Menzel 3, NGC6537, He2-320, and MyCn18. Red, green, and blue colors represent the emission from $\mathrm{N}^{+}(\mathrm{F} 658 \mathrm{~N}), \mathrm{H} \alpha(\mathrm{F} 656 \mathrm{~N})$, and $\mathrm{O}^{++}(\mathrm{F} 502 \mathrm{~N})$. For Hen2-320 $\mathrm{N}^{+}$ $(\mathrm{F} 658 \mathrm{~N})$ is depicted in orange and $\mathrm{H} \alpha(\mathrm{F} 656 \mathrm{~N})$ in cyan. (Color version online only.)

not standing shocks, to account for their structure and low ionization. These formation of low-ionization knots are yet to be successfully explained by astrophysically realistic multi-dimensional hydro accretion models of PNe.

HST images of PNe with bipolar structures are beguiling (Fig. 3). Reasonable explanations for their many structures are yet to emerge from the ongoing debate about their underlying ejection processes. HST images coupled with ground-based studies of Doppler motions show that bipolars have not formed through the ongoing interactions of evolving stellar winds. Rather, the visible material in bipolar lobes was ejected in a single relatively brief but highly organized event. This implies that bipolars are formed differently than their simpler counterparts. However, despite HST's spatial resolution of about 50 $\mathrm{AU}$ (at a distance of $1 \mathrm{kpc}$ ), its images alone do not yet allow us to probe and identify the outflow collimation mechanism(s). The images do, however, provide important spatial constraints on the models. Over time, proper motion studies will play a key role in following the trajectories of the smaller structures embedded within the outflowing gas.

It goes without saying that high-resolution imaging is HST's strongest asset for work in $\mathrm{PN}$ research. It quickly follows that imaging surveys would be of immense value. However, such surveys require time - HST's most limited resource. Even so, several surveys of nascent and infantile PNe have been conducted over the years.

Bond \& Ciardullo's snapshot survey of central stars was the first of the major imaging surveys (see above). However, their exposures were very short and the wide filters they used blended emission lines. Sahai and his collaborators (Sahai \& Trauger 1998) undertook an snapshot imaging of tiny PNe of low ionization with the goal of probing youthful PNe whose central stars have just started to ionize their winds. In addition to several small bipolars (whose ionization is often low) they uncovered a new class of PNe, multi-armed "starfish", that have no counterparts among PNe that are more highly ionized. At about the same time a group led by M. Meixner used NICMOS to image a list of pre - that is, not-yet ionized-PNe ("pPNe") to study intrinsic patterns in their dust morphologies so as to better understand the massive "superwind" ejection mechanism(s) at the tip of the AGB. The results were published in Ueta et al. (2000) Similar surveys of neutral and compact, low-ionization PNe have continued sporadically, each using 


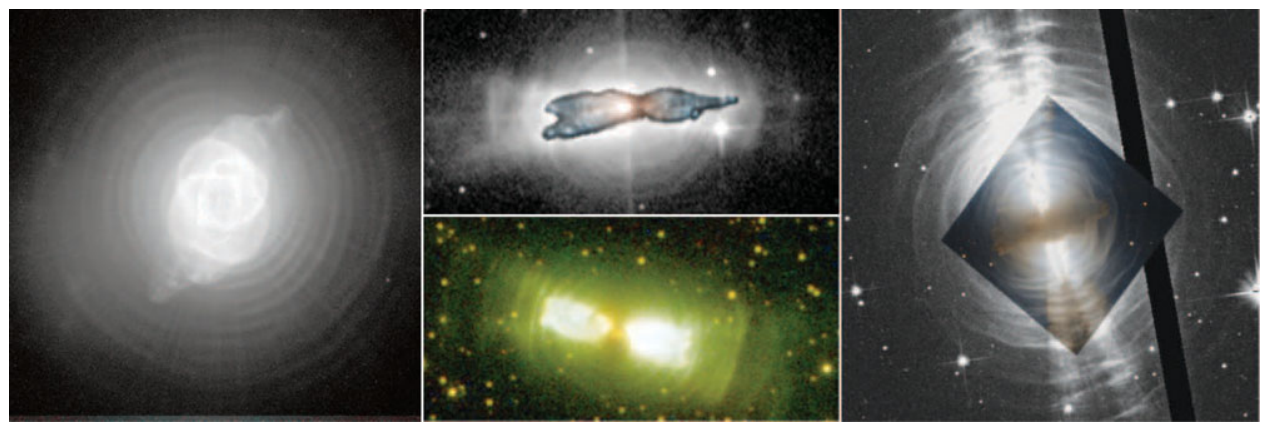

Figure 4. A montage of $\mathrm{pPNe}$ and $\mathrm{PNe}$ with outer rings. From left to right the nebulae are NGC6543, CRL618 (upper), IRAS17150-3224 (lower) and CRL2688. The images shown here were constructed by the author from images downloaded from the HST archives. NGC6543: SM3/ACS: GO9026 (Z. Tsvetanov; F502N). CRL618: GO11580 (B. Balick et al. F110W and F160W). IRAS 17150-3224: GO6364 (M. Bobrowsky; F450W and F814W) and 6565 (S. Kwok; F606W). CRL2688: GO9586 (W. Sparks; F606W) and GO11580 (Balick et al. F606W and $\mathrm{F} 814 \mathrm{~W}$ ). (Color version online only.)

different selection criteria. The results of the newest surveys (Sahai et al.(1998) GO10185; Stanghellini et al., (2009) GO11657) appear elsewhere in the proceedings of this meeting.

Concentric rings or segments (arcs) surround many PNe and pPNe. These faint features provide insight into the evolution of stars as they approach the tip of the AGB preceding the dense superwind ejections that ultimately produce the bright cores of PNe. The first rings were found in the pPN CRL2688 by Sahai et al.(1998). They are now identified in more than a dozen pPNe and PNe, examples of which are shown in Fig. 4.

HST has more-than-adequate spatial resolution to monitor changes in PNe corresponding to motions $10 \mathrm{~km} \mathrm{~s}^{-1}$ (the sound speed of gas at $\sim 10^{4} \mathrm{~K}$ ) over a decade at distances out to $2 \mathrm{kpc}$. CRL2688 is one of a growing number of examples Balick et al.2012). The measured angular growth rate of its rings, 0.07" in $6.5 \mathrm{yr}$, coupled with $\mathrm{CO}$ absorption observations (Bujarrabal et al. 2001, Fong et al. 2006) show that their outflow speed is $18 \mathrm{~km} \mathrm{~s}^{-1}$. This result is in very close agreement with the predicted terminal velocity of radiation-drive dust winds in AGB stars by Ivezić \& Elitzur (2010). Multi-epoch proper motion studies will prove to be a valuable new and new "movie camera" of proper motions in pPNe and PNe that extends our empirical understandings into the time domain.

Hubble's great suite of cameras, from WFPC2 and NICMOS in the early years to WFC3 today, has enabled comparative "color" studies of structures from 0.2 to 2 microns and far beyond. This is particularly valuable for studies of the geometries of molecular and the many ionic species of ionized gas. (WFC3 with its broad array of many narrowband filters is an especially powerful tool.) And, of course Chandra, XMM, and a variety of mid-IR and radio interferometers produce images with comparable spatial resolution and PSF quality that make HST images critical templates for the comparison of structures and their overall morphology.

The first of such comparisons appeared for emission lines of ionized gas in NGC6543 in 1995 (see above; Fig 1 left) and for molecular lines and dust in CRL2688 in 1997 (Thompson et al. STScI press release 1997-11). The latter study showed that $\mathrm{H}_{2}$ arises at the periphery of the two bright polar reflection lobes and an opaque equatorial dust lane in the core of this pPNe. Another spectacular case is the overlay of NICMOS IR continuum, $\mathrm{H}_{2}$, and visible light images for NGC7027 (Latter et al. 2000a). Similar IRoptical overlays were made for Hubble $12, \mathrm{BD}+30^{\circ} 3639$, and $\mathrm{OH} 231.8+04.2$ (Bieging et al. 2000; Hora et al. 2000; \& Latter et al. 2000b). X-ray images from Chandra and 
XMM have also been used in conjunction with HST images to show that the extended $\mathrm{X}$-rays arise inside closed bubbles with sharp rims that are centered on the central star.

The unique optical and ultraviolet spectroscopic capabilities of HST/STIS have been used to study complex nebular structures and the nuclear enrichment processes in highly evolved stars. A comparative study of relative C, N, O, and Ne abundances in the LMC was made by Stanghellini et al. (2005) and extended to the SMC by Stanghellini et al. (2009) Their comprehensive work shows that the theoretical models that describe the enrichments of $\mathrm{C}$ and $\mathrm{N}$ in AGB stars of various masses are generally verified observationally. A variety of optical and ultraviolet studies of individual PNe have also appeared. Palla et al. (2002) used STIS to measure the isotopic rations of ${ }^{12} \mathrm{C}$ and ${ }^{13} \mathrm{C}$ from the bright CIII] doublet lines of NGC3242. Rubin et al. (2002) searched for small-scale temperature fluctuations within NGC7009. Perinotto et al. (2004) analyzed long-slit STIS observations to study changes in temperature and density of the bright PN NGC7662 at subarcseond resolution. Two additional spectroscopic programs have been approved for HST Cycle 19. One is a search for the lines expected to arise at the interface of the hot X-ray bubble $\left(>10^{6} \mathrm{~K}\right)$ and the surrounding warm $\left(\sim 10^{4} \mathrm{~K}\right)$ nebular gas in NGC6543 (Guerrero et al. 2001). Another is a survey of the CNO abundance ratios in ten selected Galactic PNe (Dufour 1991). These programs illustrate the potential scientific power of HST's spectrographic instruments.

In summary, HST has been an astronomical microscope, revealing details of PNe that are upending some old, cherished beliefs, provoking many new ideas, and making it possible to add critical constraints on theoretical studies. We're using it to peer into the wombs of PN birth and cutting through all but the most severe extinction using near-IR cameras on HST. HST is also our only window into the uv spectrum where critical carbon lines (not to mention many others) are uniquely accessible, and we can complement our images using long-slit spectroscopy at very high spatial resolution to probe the details of the changes in physical conditions in or near shocks.

For example, the idea that magnetic fields may structure wind outflows is based, in part, on the complexity of bipolar pPNe and PNe revealed in HST images. As a result the effort is underway to develop full 3-dimensional MHD models with radiation transfer in a rotating coordinate frame - truly a Grand Challenge - has been provoked by the combination of Hubble with complementary types of data. This model will be essential to model mass transfer and outflow in close binaries and, hence, to probe the origins of highly collimated superwinds. Such physics can be extended to many classes of astrophysical objects, from cataclysmic variables to merging galactic nuclei.

The last suite of instruments have now been installed on HST. At some point the gyros, batteries, or some critical control unit will fail and HST's useful life ends, perhaps as early as 2014. It will not be replaced with another telescope with its extremely capable filter complement and spatial resolution. So the central issue is how to optimize the use of HST's remaining days. Here we are limited only by our imagination, competition from other astronomers, and a little good fortune.

In late 1995 Mario Livio adeptly and presciently summarized the science challenges for research in PN (and closely related nebulae) at a conference devoted to the scientific potential of HST (Livio 1996). Since then, and led by HST images, our observations have multiplied in depth and breadth. To judge from conference attendance and presentations, interest, information, and excitement are growing steadily. Still, the high-level scientific challenges outlined by Livio and elucidated by Balick \& Frank (2002) remain much the the challenges in 2011. Obviously those challenges have turned out to be formidable. Nonetheless they remain important: late mass ejection from low-mass stars is turning out to be a very tricky piece to place in the puzzle of stellar evolution. 


\section{References}

Balick, B. \& Frank, A. 2002, ARAA, 40, 439

Balick, B., Gomez, T., Vinković, D., Lacolea, J., Corradi, R. L. M., \& Frank, A. 2012, ApJ, 745,188

Bieging, J. H., Meakin, C. A., Kelly, D. M., Dayal, A., Latter, W. B., Hora, J. L., \& Tielens, A. G. G. M. 2000, Asymmetrical Planetary Nebulae II: From Origins to Microstructures, 199,183

Bujarrabal, V., Castro-Carrizo, A., Alcolea, J., \& Sanchez Contreras, C. 2001, A\&6A, 377, 868

Ciardullo, R., Bond, H. E., Sipior, M. S., Fullton, L. K., Zhang, C. Y., \& Schaefer, K. G. 1999, $A J, 118,488$

Dufour, R. J. 1991, PASP, 103, 857

Fong, D., Meixner, M., Sutton, E. C., Zalucha, A., \& Welch, W. J. 2006, ApJ, 652, 1626

Guerrero, M. A., Chu, Y. H., Gruendl, R. A., Williams, R. M., \& Kaler, J. B. 2001, ApJL, 553, L55

Hora, J. L., Latter, W. B., Dayal, A., Bieging, J., Kelly, D. M., Tielens, A. G. G. M., \& Trammell, S. R. 2000, Asymmetrical Planetary Nebulae II: From Origins to Microstructures, 199, 267 Ivezić, Ž. \& Elitzur, M. 2010, MNRAS, 404, 1415

Latter, W. B., Dayal, A., Bieging, J. H., Kelly, D. M., Hora, J. L., \& Tielens, A. G. G. M. 2000, Asymmetrical Planetary Nebulae II: From Origins to Microstructures, 199, 263

Latter, W. B., Dayal, A., Bieging, J. H., Meakin, C., Hora, J. L., Kelly, D. M., \& Tielens, A. G. G. M. 2000, ApJ, 539, 783

Livio, M. 1996, Science with the Hubble Space Telescope - II, 257

Palla, F., Galli, D., Marconi, A., Stanghellini, L., \& Tosi, M. 2002, ApJ(Letters), 568, L57

Perinotto, M., Patriarchi, P., Balick, B., \& Corradi, R. L. M. 2004, A\&\&A, 422, 963

Bhatt, N. J., Dufour, R. J., Buckalew, B. A., et al. 2002, MNRAS, 334, 777

Sahai, R. \& Trauger, J. T. 1998, AJ, 116, 1357

1998ApJ...493..301S Sahai, R., Trauger, J. T., Watson, A. M., et al. 1998, ApJ, 493, 301

Stanghellini, L., Lee, T. H., Shaw, R. A., Balick, B., \& Villaver, E. 2009, ApJ, 702, 733

Stanghellini, L., Shaw, R. A., \& Gilmore, D. 2005, ApJ, 622, 294

Ueta, T., Meixner, M., \& Bobrowsky, M. 2000, ApJ, 528, 861 